\title{
Monell Chemical Senses Center
}

A taste of the research at the Monell Center reveals an increased understanding of how we perceive and integrate chemical cues.

When biophysicist Joseph Brand teamed up with geneticist Xia Li and several colleagues to report that cats do not express Tas $1 r 2$, they could not have predicted how the public would respond. This gene encodes T1R2, or one half of the T1R2/T1R3 sweet receptor, without which it should be impossible for cats to sense that a food is sweet. Although this discovery was in agreement with previous circumstantial evidence, cat owners across the country were indignant, each testifying to the fact that his or her cat did indeed enjoy eating sweets. Though the origin of these marshmallow-eating cats remains a mystery, the origin of the research stems from a strong commitment to understanding the molecular and genetic bases for taste and smell at the Monell Chemical Senses Center.

The Monell Center (www.monell.org/) was founded in 1968 in Philadelphia, Pennsylvania with the support of the Ambrose Monell Foundation, and for its first ten years was associated with the University of Pennsylvania. Since the beginning, the central goal of the institute has been to explain the chemical senses, from elucidating the physical basis for detecting chemicals in the nose and mouth, to deconvoluting the wiring that converts these chemicals to signals in the brain, to the further processing of those signals that tells us that we're sated, or that we've smelled freshly mown grass. Put another way, Director Gary Beauchamp, a biopsychologist, says "we're trying to make connections not just logical but mechanistic." To accomplish this goal, the Monell Center currently houses about $60 \mathrm{PhD}$-level scientists from a wide range of fields including genetics, psychology and neurobiology. Though coordinating scientists from such disparate backgrounds could present hurdles in communication, Charles Wysocki, a psychobiologist, says having a single overarching focus promotes "a free flow of information across disciplines."

The interdisciplinary and collaborative atmosphere is reflected in the ongoing research projects. Rather than having formal research groups, particular projects seem to grow from the common interests of several Monell scientists. Maintaining a diverse faculty then naturally spurs new directions and opportunities at the intersection of individual expertise. For example, Danielle Reed, who was trained as a psychologist, is forming biological links between phenotype and genotype, or between perception in the brain and the complement receptors. Kai Zhao, a bioengineer, envisions the nose as a gas chromatograph, with different regions having different flow speeds to compensate for the varying physical properties of inhaled chemicals. Computational models of these regions then provide insight into how the flow speed might be controlled. Alan Gelperin, a neuroscientist, is trying to understand how odor information is processed in the brain, leading to long-term odor memory storage. As our understanding of this and related processes improves, he sees the day when an artificial olfactory system will come to fruition. In contrast, Brand is keenly aware of how far the field has already come: he remembers, when he started at the institute in 1972, how "we were forced to defend the receptor hypothesis," or the idea that exogenous chemicals are directly sensed by discrete protein receptors.

Both the science and the physical surroundings have made strides since the early days, with recent renovations and a significant expansion allowing the Monell faculty nearly free reign in designing their laboratories. The two spacious buildings that the center now occupies house organic chemistry labs, molecular biology rooms and human testing facilities. The particular organization of the labs throughout the buildings serves to promote open communication. Mark Friedman, who was trained in physiological psychology, notes that as faculty make their way from one lab to another, "a lot of science gets done in the hallways."

The diverse lab space and organization also allow Monell scientists to follow a single train of scientific thought through various levels of chemical and biological complexity. For example, as Beauchamp points out, researchers can conduct "molecular and sensory biology experiments on the same person," a key factor in determining the genetic basis of a sensory profile. With this kind of data available, Monell researchers are able to pursue a variety of sensory puzzles. For example, George Preti, who was trained as an organic chemist, is making inroads into understanding what chemicals humans make; in particular he is seeking to identify chemicals involved in the link between body odor and

\begin{tabular}{l} 
"We're trying to make \\
connections not just \\
logical but mechanistic." \\
- Gary Beauchamp \\
\hline
\end{tabular}

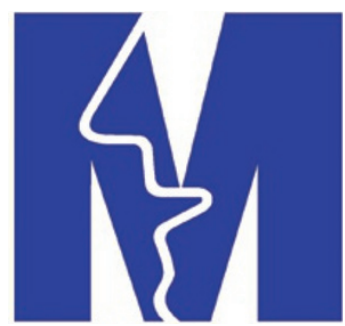

major histocompatibility complex genes. Wysocki is curious to know why people with specific anosmias can't respond to a particular chemical at all, and how our sensitivity to chemicals can change over time. His interest in the topic undoubtedly stems from his own early specific anosmia, which prevented him from distinguishing the smell of lemonade from that of skunk.

With several ongoing research programs, the Monell faculty is reliant on the general public for willing volunteers. The Monell-Jefferson Taste \& Smell Clinic, a joint venture with Thomas Jefferson University, provides an additional opportunity to engage with the public in providing diagnoses of smell and taste disorders. Scientists at Monell also maintain collaborations (and some even hold positions) with their historic partner, the University of Pennsylvania. Though Monell is an institute rather than a university, and thus has no graduate program, students remain a common sight at Monell, with both intensive eight-week summer internships and year-round opportunities for undergraduate and high school students, as well as the occasional graduate student (in partnership with neighboring schools). These interactions with members of the public and with the scientific community highlight the commitment of the Monell staff to their overall mission "to advance scientific understanding of the mechanisms and functions of the chemical senses to benefit human health and well-being."

The research at the Monell Chemical Senses Center reflects the significant advances in the field in the last four decades, when, as Brand says, “we didn't know squadoodle about taste." And yet significant mysteries remain. The sour and salty receptors have yet to be definitively identified. The events that allow one cell recognizing a chemical stimulus to force an adjoining cell to report that event to the brain are unclear. And the marshmallow-eating cats suggest that there may be more to learn about tasting sweets than T1R2. With all these topics to explore, hopefully the team at Monell is hungry for a challenge.

Catherine Goodman, Philadelphia, Pennsylvania 